\title{
Experiment and Simulation Study on the Gear Tooth Modification of Helical Gear Pair based on Dynamic Performances
}

\author{
*Ruiliang Zhang and Tie Wang \\ Gear Research Institute, College of Mechanical Engineering, Taiyuan University \\ of Technology, Taiyuan, 030024, China \\ rl_zhang@163.com
}

\begin{abstract}
The gear dynamic responses such as vibration and noise in gear transmission systems are mainly caused by transmission error (TE) excitation. Gear tooth modification (GTM) is a common approach to reducing the peak-to-peak TE and hence vibration response. GTM determination should take into account of the particular condition of the gear set. In this paper, in order to evaluate the effect of given GTM parameters on vibration and noise in a helical gear pair, comparison experiments for the gear pair with and without GTM were carried out. Simulation analysis was also employed to further illustrate the GTM affections. Both experiment and simulation results show that the given GTM parameters are ineffective on improving dynamic performance under the operating conditions that the gear is designed for. Therefore, a new GTM design are proposed, in which inevitable meshing misalignments are also accounted. Simulation analysis shows that the new GTM design exhibit a much smaller peak-to-peak TE than the old design and the baseline, showing that it is more effective in improving transmission performance. In addition it also shows that simulation analysis is an effective approach to evaluate the influence of GTM in the design stage to meet the desired dynamic performance.
\end{abstract}

Keywords: Helical gears, Gear tooth modifications (GTM), Simulation analysis, Transmission error (TE), Load distribution, Mesh misalignment.

\section{Introduction}

Helical gears are widely used in automotive, aerospace and many other industrial power trains. With the increasing demands of better dynamic performance in modern gear systems, the reduction of vibration and noise becomes increasingly important. In practice, due to the variation of meshing positions, the errors inevitably produced by the process of manufacture and assembly [1], the elastic deformation of gear teeth and the deflections of the entire system. Therefore, gears will generate irregular motion transfer and fluctuating transmission error (TE) [2], which result in high vibration and noise transmitted to the housing.

As a common approach to decreasing TE, gear tooth modifications (GTM) has been widely used to optimize contact patterns and stresses, to compensate for meshing misalignment and to reduce gear vibration and noise as well[ $[3,4,5]$.

In the process of GTM design, the fundamental and the key issue is the determination of the modification types and their corresponding parameters under given operating conditions such as variations in load and misalignment.

Nowadays, there are not uniform formulas to estimate the modification amount, although there are many methods and standards to determine the GTM parameters [5]. Generally, many enterprises select GTM parameters according to their own empirical method. "Typical traditional approach" to gear tooth modifications design refines microgeometry by experience/experiment and repeated prototyping [6]. An experimental study 
is the most effective approach to evaluate the effect of GTM on vibration reduction, and many experiments were performed to analyze the effect of the GTM on vibration [7-10]. Obviously, this method is at high economical and time cost. With the development of high-performance computers, numerical models and finite element analysis (FEA) models have become popular for studying the modification parameter and its effect on the gear performance [1,7,11-17]. These studies provide an approach to studying the dynamics of gear meshing process, although they did not study the effect of different GTM methods and its corresponding parameters on TE. In addition, the above-mentioned studies did not provide any negative examples to indicate the agreement between simulation results and experiment ones for the relationship of TE and vibration.

In this paper, comparison experiments of the helical gear pairs with and without modification are carried out under its operating conditions in order to validate the effect of GTM on vibration and noise. The experimental results show that the RMS of vibration signals and noise value of modified gear pairs increase obviously. In order to find out the reason that caused this results and to propose corresponding measures, a simulation analysis of modified and unmodified gear pairs is introduced to evaluate the effect of the GTM on TE based on a commercial software package. The analysis results indicate that there is good agreement between TE and tested vibration. Then mesh misalignment under working load is considered in this paper for GTM determination. The new GTM method and parameters are proposed based on mesh misalignment and standard formula, and its effect on TE is validated.

\section{TE Theory and Mesh Misalignment}

\subsection{TE Theory}

It is now accepted that the noise and vibration levels correlate well with TE. TE is a deviation of the position of the driven gear from its theoretical one (which it would occupy as defined by the gear ratio if both gears were geometrically perfect and infinitely stiffness) [18]. It is calculated using the micro-geometry of the gears and the misalignments in each loaded condition [19]. When a pair of gears has ideal profiles running with no loading, theoretically, they should mesh with zero TE. But actually, considering the manufacturing errors, gear tooth deflections, shaft deflections and mesh stiffness variability, TE will not keep constant as zero. It can be expressed as either angular value or linear value. In this paper, TE is expressed as a linear value represented by Eq. (1).

$$
T E=r_{b 2}\left(\theta_{2}^{\prime}-\theta_{2}\right)
$$

where $r_{b 2}$ is base radius of driven gear; $\theta_{2}$ and $\theta_{2}^{\prime}$ are the theoretically angular value and the actual one of driven gear, respectively.

\subsection{Mesh Misalignment}

Mesh misalignment is one of the main factors that cause TE. Generally, when mesh misalignment happening, the gear mesh occurs on one end of the face width and the deflection of gears under load on the other end will appear. And the biggest value of this deflection is defined as meshing misalignment, which is illustrated in Figure 1. The meshing misalignment can cause an uneven load distribution and unstable transmission. The misalignment is an important factor that should be considered when adopting suitable modification method. 


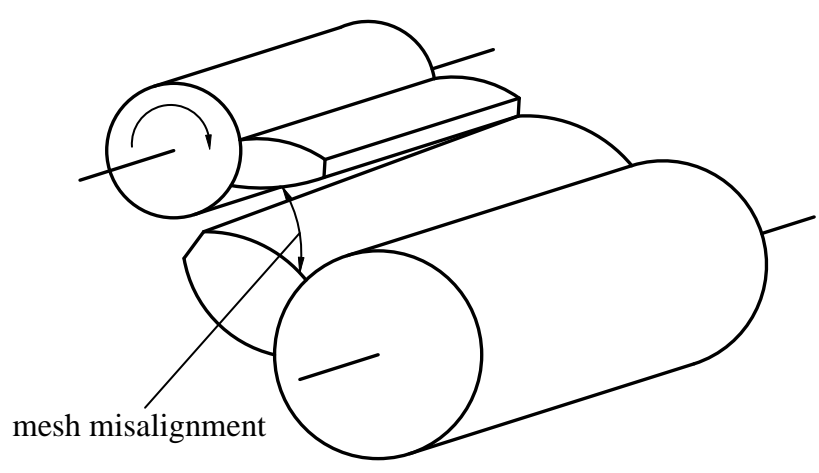

Figure 1. Illustration of Mesh Misalignment [20]

\section{Gear Tooth Modification}

GTM, so called micro-geometric modification, indicates the intentional removal of material from the gear teeth flanks so that the resulting shape is no longer a perfect involute. Generally, GTM can be classified as 1) profile modification and 2) lead modification.

\subsection{Profile Modification}

The profile modification changes the tooth profile shape with a perfect involute curve. Profile modification can improve effectively the phenomenon of load varying substantially, lower the meshing impact and vibration caused by the elastic deformation of gear teeth and assembly errors of the gear pairs, and reduce gear dynamic excitations.

Profile modification can be classified several types such as tip relief, root relief, profile crowning, or any defined gear flanks topography. As root relief can result in weakening root strength, the tip relief is adopted as a popular profile modification measure. In the case of this study, it is tip relief with a parabolic curve (as shown in Figure 2). Tip relief is determined by three key parameters: modification amount $\Delta$, modification length $h$, and the modification curve.

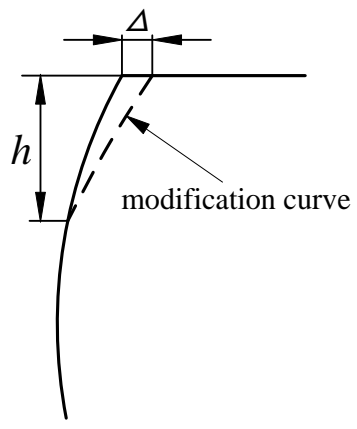

Figure 2. Tip Relief Modification

\subsection{Lead Modification}

Lead modification indicates the intentional modification performed along the longitudinal direction. Lead modification plays an important role in avoiding partial load, improving tooth load distribution and TE. There are several types of lead modification such as lead crown, end relief, and lead slope modification. Lead crown or end relief is often used to compensate for mesh misalignment so that the loads and hence stresses, do not rise at the tooth edges and TE is smoothened. 
In this paper, the comparison of end relief and crown affecting the TE will be carried out.

3.2.1. End Relief: End relief is the slightly chamfering of single end/both ends of the tooth flanks. It is used to avoid edge contact caused by contact deformation under slight misalignment. Usually, the end relief applied is the same at both ends of the teeth, as shown in Figure 3. The amount of $c_{c}$ and the length $b_{c}$ are used to determine the end relief.

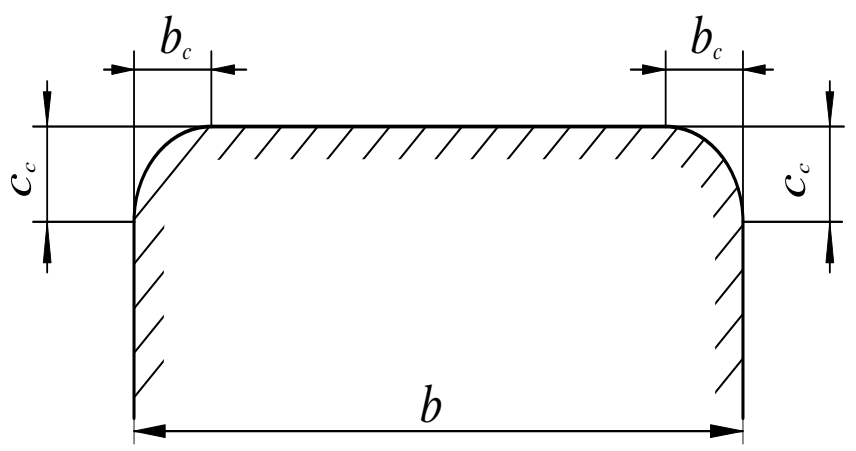

Figure 3. End Relief

3.2.2. Lead Crown: Lead crown is the removal of a slight amount of tooth flank from the center to edge, making the tooth flank slightly convex. Gears are usually crowned symmetrically about the mid-face width. Lead crown allows a greater tolerance in the misalignment than end relief. The crown amount should not be larger than necessary as otherwise, it would reduce dimensions of tooth contact, thus increasing transmission error. The crown amount $\mathrm{Cc}$ of gear is a key parameter, which influences the effect of lead crown significantly [22], as shown in Figure 4.

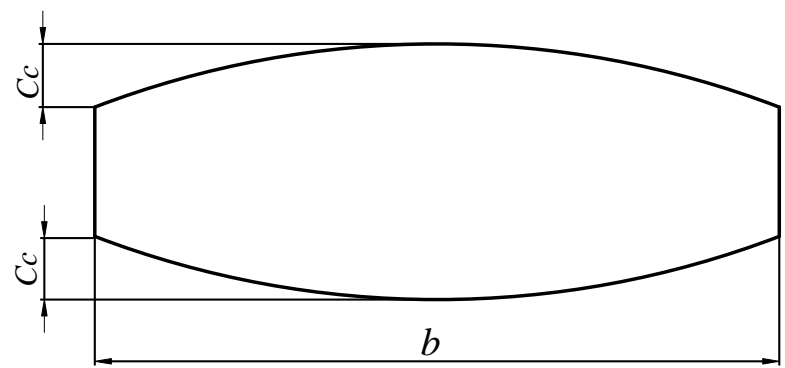

Figure 4. Lead Crown

\section{Experimental Verification}

In order to validate the effect of the given modification on dynamic performance under working condition, a comparison test was carried out by measuring vibration and noise between two sets of identical gears specified in Table 1 are identical but one of them with tooth modification, detailed in Table 2, and the other is without any tooth modification. Both gear sets were tested under their designed operating conditions: a constant torque of $600 \mathrm{Nm}$ at a constant speed of $1200 \mathrm{rpm}$ on an in-house gearbox test system shown in Figure 5.

To evaluate their dynamic performance, vibration signals were measured by four accelerometers mounted on the bearing seat of the gearbox on both radial and axial directions. In the meantime, noise signal is also measured by a wideband B\&K microphone. All the data were logged by a multiple-channel, high-speed data acquisition 
system with $25.6 \mathrm{kHz}$ sampling rate and 16-bit resolution. Each test was carried out for 4hours, the data were collected for 1 minutes at an interval of 30min.

\section{Table 1. Geometric Parameters of Helical Gear Pairs}

\begin{tabular}{|l|l|l|}
\hline Parameters & Pinion & Gear \\
\hline Number of teeth & 18 & 33 \\
\hline Face width (mm) & 36 & 34 \\
\hline Profile Shift coefficient & 0.2750 & 0.3083 \\
\hline Normal module (mm) & $5 \mathrm{~mm}$ \\
\hline Normal pressure angle (deg.) & 20 \\
\hline Helix angle (deg.) & 30 \\
\hline Precision grade & 6 \\
\hline Center distance (mm) & 150 \\
\hline Transverse /axial contact ratio & $1.22 / 1.082$ \\
\hline Total contact ratio & 2.302 \\
\hline
\end{tabular}

Table 2. Gear Modification Parameters

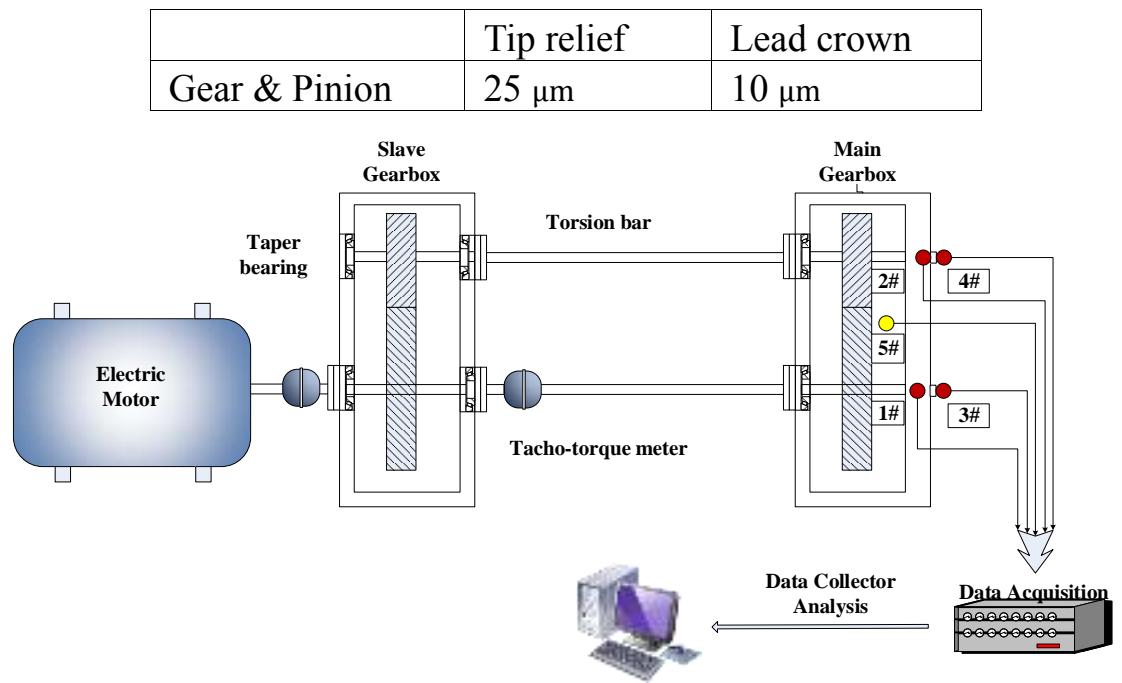

Figure 5. Schematic Diagram of Test Rig

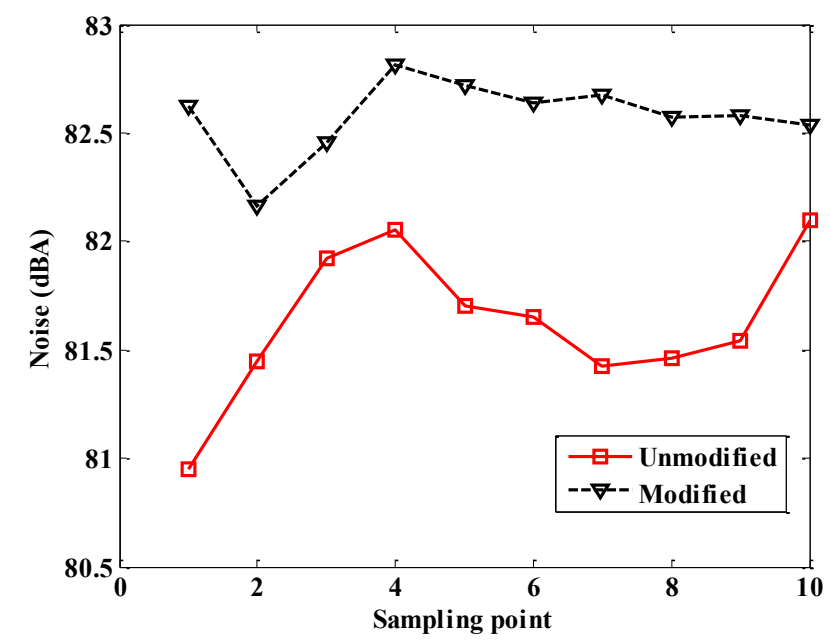

Figure 6. Comparison of Noise between Modification and Non-modification Gear Pairs 
Ten sets of test data have been chosen evenly from the acquired data. The comparison results of noise and RMS of the collected data for modified and unmodified gear pairs are represented in Figure 6 and Figure 7, respectively. And the averaged values of these ten sets for comparison are listed in Table3. The results presented in Table 3 indicate that the effect of the modification on overall noise increases by $1 \%$. The RMS values of vibration on all four accelerometers for modification increase obviously compared with nonmodification. The maximum value increase by $23 \%$ in case of modification, as shown in Table 3.

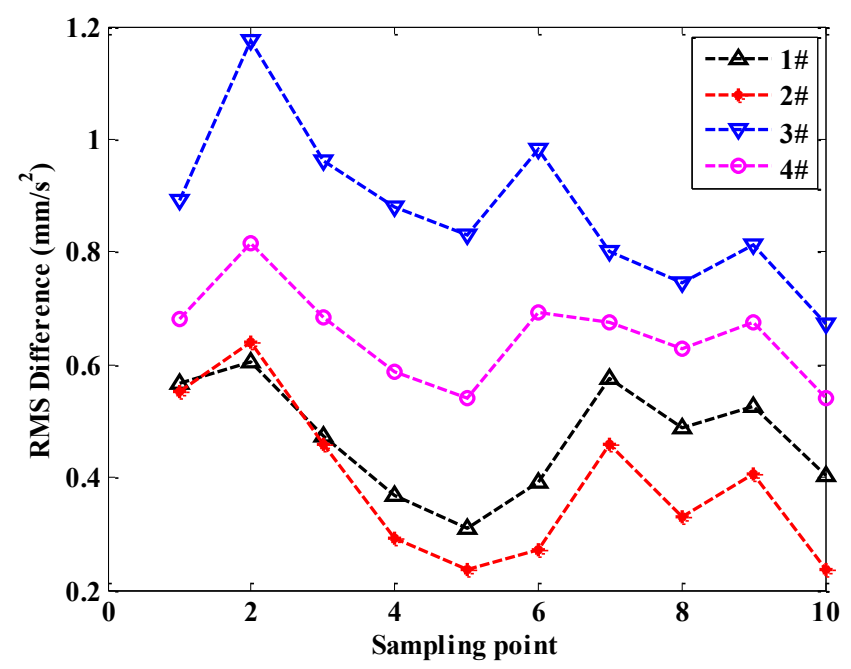

Figure 7. Comparison of RMS of Vibration Signals between Modified and Unmodified Gear Pairs

Table 3. The Average Value of Noise and RMS Value of Vibration Signals for Modified and Unmodified Gear Pairs

\begin{tabular}{|l|c|c|c|c|}
\hline & No. of Sensor & $\begin{array}{l}\text { unmodified } \\
\text { (Baseline) }\end{array}$ & Modified & Increase (\%) \\
\hline \multirow{3}{*}{ RMS $\left(\mathrm{mm} / \mathrm{s}^{2}\right)$} & 1 & 3.26 & 3.73 & 14.4 \\
\cline { 2 - 5 } & 2 & 3.51 & 3.90 & 11.1 \\
\cline { 2 - 5 } & 3 & 3.80 & 4.68 & 23.0 \\
\cline { 2 - 5 } & 4 & 3.76 & 4.41 & 17.3 \\
\hline Noise (dBA) & 5 & 81.56 & 82.55 & 1 \\
\hline
\end{tabular}

The results show that noise and vibration of the given modified gear pairs are larger than unmodified ones. It indicates that the presented modification is not effective on noise and vibration reduction around the working condition $(1200 \mathrm{r} / \mathrm{min}, 600 \mathrm{Nm})$, whereas it results in worse dynamic behavior. The reason is that the modification method or modification amount is not suitable for presented working condition. A further comparison will be made with the simulation results in the next section for explaining the experimental results. 


\section{Simulation Analysis}

In this paper, the model of the gear pairs is constructed, as shown in Figure 8 [22]. Then TE and load distribution for modified and unmodified gear pairs are compared based on simulation results.

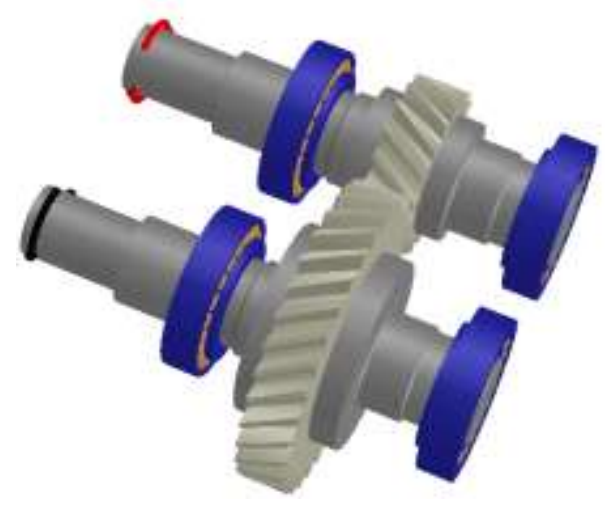

Figure 8. Model of Gear Pairs

\subsection{Signal Processing Results}

5.1.1. TE Comparison: The TE curves of three meshing cycles are shown in Figure 9. Due to the meshing stiffness varies significantly in alternation process, the TE fluctuates during the alternation process. Here the movement smoothness is concerned, that means less fluctuation can result in more smoothness. The peak-to-peak TE of modified gear is $3.49 \mu \mathrm{m}$ which is larger, about 3 times than $1.23 \mu \mathrm{m}$ of unmodified ones.

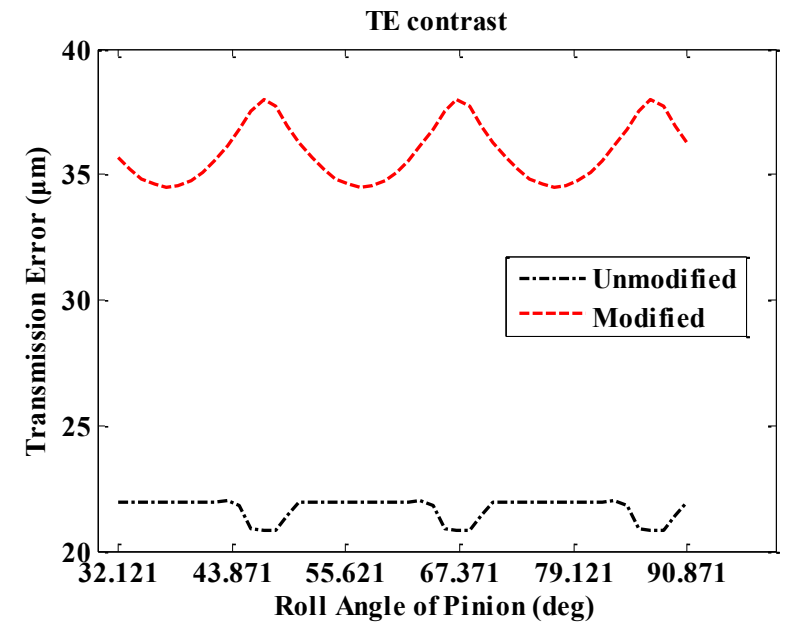

Figure 9. The TE of the Pinion

5.1.2. Load Distribution Comparison: It can be observed from Figure 10 (a) that the load is distributed unevenly along the face width. The red parts of the figure indicate the high load acting on the gear tooth flank. The phenomena of edge contact and tooth partial load are obvious.

The contact load distribution for modified is shown in Figure 10 (b). It shows that the design has centered the contact, edge contact has been avoided, but load distribution still is uneven along the face width. The peak load for modified is $549 \mathrm{~N} / \mathrm{mm}$, which is $78 \%$ higher when compared with the unmodified result due to the reduction in contact area caused by more centralized contact across the load range. 

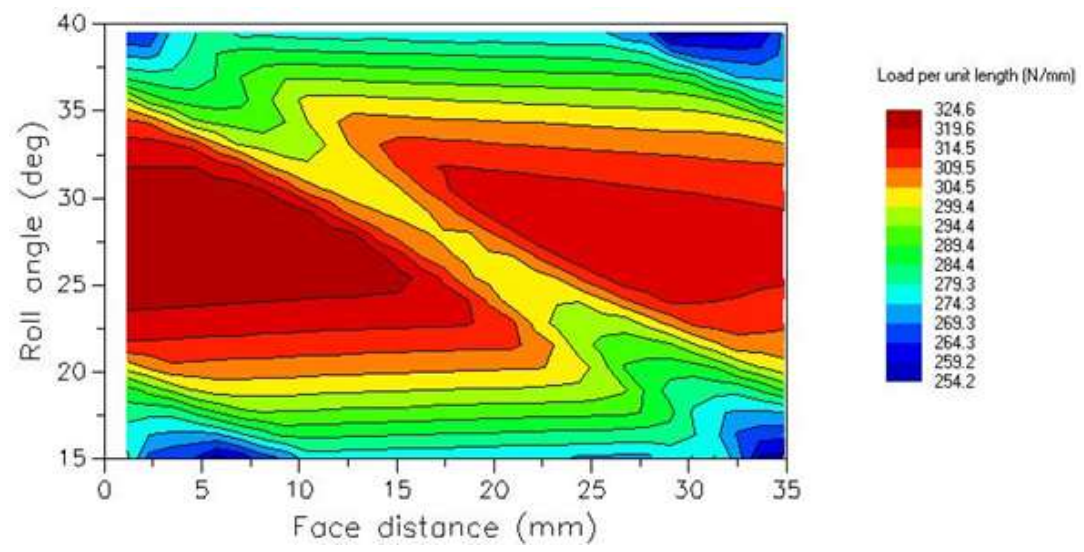

(a) Ummodified

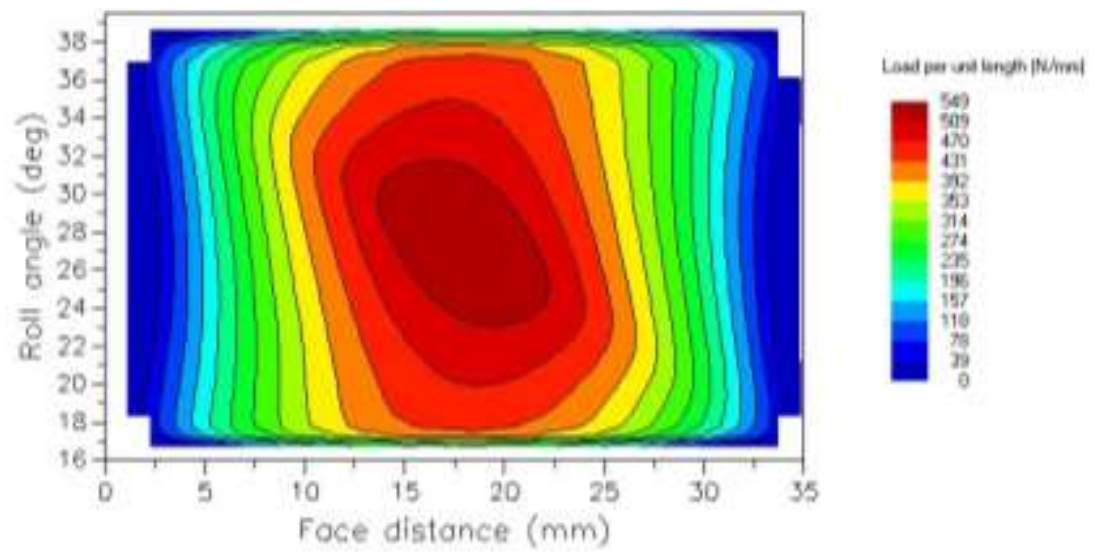

(b) Modified

Figure 10. The Load Distribution of the Pinion

\subsection{Discussion}

Both the simulated and experimental results show that the apparent "success" of the adopt approach and amount of the GTM is only valid for edge contact avoided. Far from being reduced, the noise level and RMS of vibration at the working condition increase $1 \%$ and $23 \%$ respectively. This reveals that the presented modification is not "successful" at all. And it also can be concluded that obviously increased TE results in the worse dynamic feature of modification gear. The further and detailed investigation should be made to design modification and improve the performance.

\section{The New GTM Design and Simulations Results}

\subsection{Mesh Misalignments Analysis}

Gear mesh misalignment of studied gear pairs is considered in this paper as shown in Figure 11. And the combined deflection between two gears is $0.7895 \mu \mathrm{m}$. As mentioned above, the lead crown modification is not suitable at the condition of the slight mesh misalignment. That is why the above-mentioned modification did not reduce the TE. Edge relief is superior to lead crown under slight misalignment [23], therefore end relief is employed in new GTM design. 


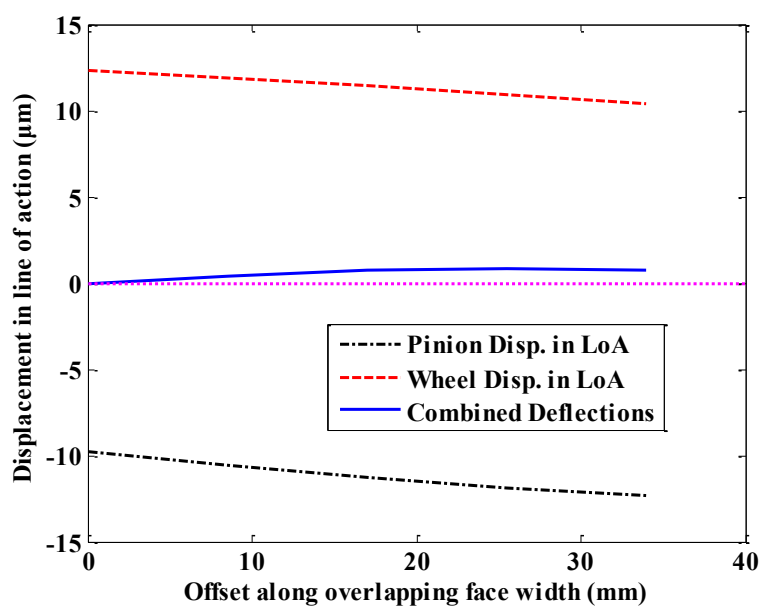

Figure 11. Mesh Misalignment

\subsection{Simulation of New GTM Design}

The profile modification still is tip relief, and its parameters are same as that shown in Table 2.

6.2.1. Determination of End Relief Amount: The formula of end relief amount $c_{c}$ is expressed as follows [23]:

$C_{c}=\frac{F_{m}}{b C_{\gamma}}$

where $C_{\gamma}$ is comprehensive meshing stiffness, $b$ is gear tooth width, $F_{m}$ is mean transverse tangential load.

Generally, the maximum tooth deformation appears at the position where the distance from both ends of tooth flanks ranges between $7 \%$ and $8 \%$. At this position, there is quite obviously edge contact effect and the maximum contact stress. In order to avoid stress concentration, the length of end relief $b_{c}$ should be larger than above-mentioned distance and is expressed by:

$b_{c}=0.1 b$

where: $b$ is tooth face width (see in Figure 3).

Taking into account of misalignment and working condition of gear pairs, the parameters of end relief with a parabolic curve are determined as follows: $b_{c}=3.4 \mathrm{~mm}$, and $\mathrm{C}_{\mathrm{c}}=24 \mu \mathrm{m}$.

The end relief parameters are shown in Figure 12 and applied to both gear and pinion.

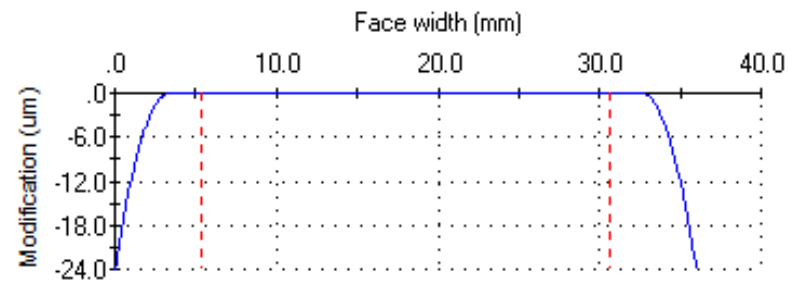

Figure 12. End Relief 
6.2.2. Simulation of End Relief Gear: The simulation results of end relief pinion at working condition are shown in Figure 13.

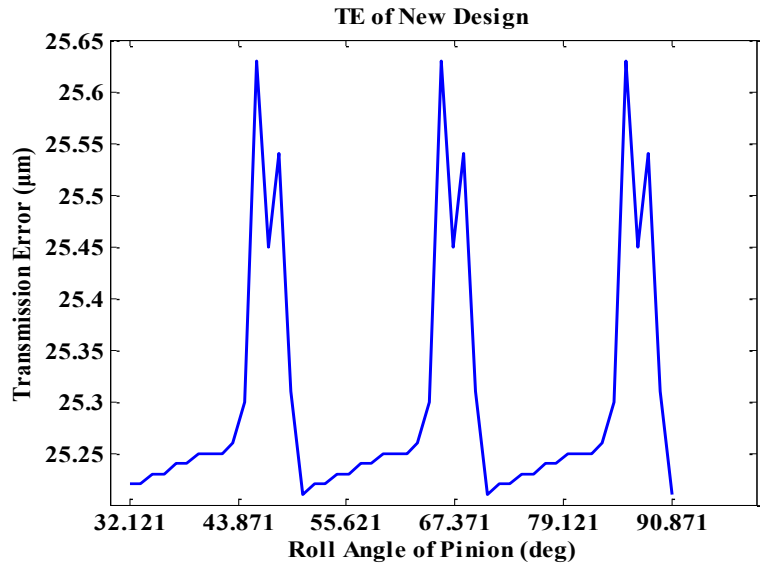

(a) TE of New Design

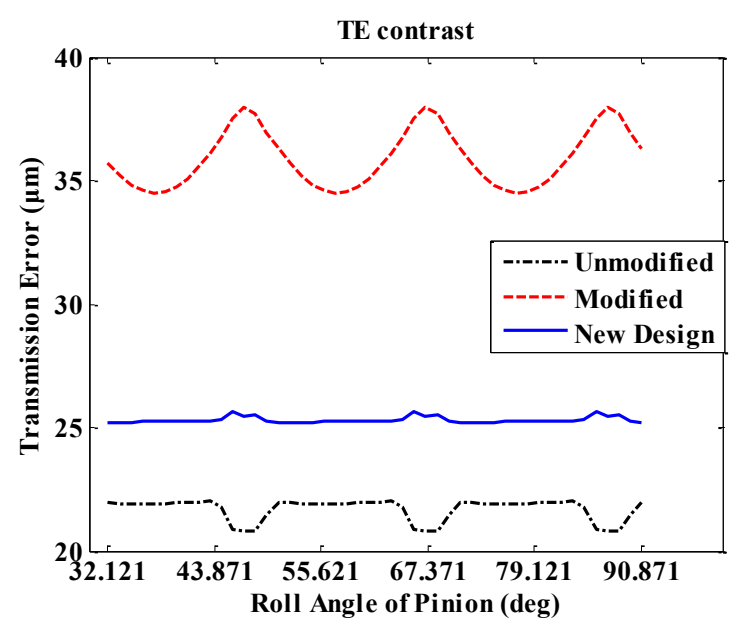

(b) TE Comparison

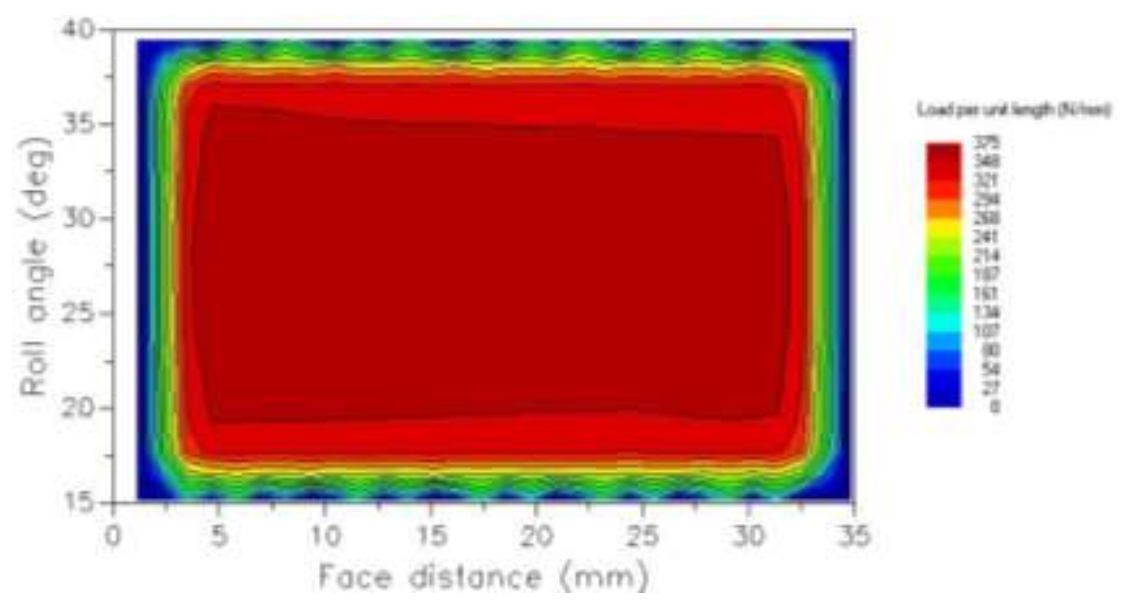

(c) Contact Load Distribution

Figure 13. The Analysis Results of Pinion with New GTM Parameters

Figure 13 (a) shows that the peak-to-peak TE is $0.42 \mu \mathrm{m}$. It is obvious that new GTM design is a better choice by comparing these three modifications, detailed in Table 4. Figure 13 (c) shows a further improvement in the sense that the contact area is more even 
along the face width. The phenomenon of gear partial load disappear and the contact pattern is good across the load range which is helpful to enhance load capacity and lifetime. There is a slight increase in the peak load due to the reduction in contact area caused by the end relief. But since the TE is already very low for a transmission of this type, the new GTM design is better overall.

Table 4. Comparison of TE Fluctuation for Different Modification

\begin{tabular}{|c|c|c|c|}
\hline & Baseline & Crown & End relief \\
\hline Peak-to-Peak TE $(\mu \mathrm{m})$ & 1.23 & 3.49 & 0.42 \\
\hline Change $(\%)$ & - & 183.7 & -65.9 \\
\hline
\end{tabular}

Compared with Figure 9 and Figure 10, it can be concluded that the end relief is more effective than lead crowning on both TE deduction and contact load distribution in studied gear pairs. The end relief is more suitable for interested gear pairs under its working condition.

\section{Summary and Conclusion}

In order to validate the effect of given GTM on vibration and noise of gear set at its working condition, the comparison experiments of helical gear pairs with and without modification were carried out. The experimental results show that modified gear pairs have worse dynamic characteristics than unmodified ones. Then, the simulation analysis of modified and unmodified gear pairs is employed in order to find out the reason that caused this results and to propose corresponding measures. Based on the simulation results, the new GTM design is proposed and analyzed. The results of the simulations are compared for verification. From the results presented in this article, the following conclusions can be drawn.

1) It is important to consider the working conditions and mesh misalignment when determining the appropriate tooth modification method and parameters. The GTM method affects TE significantly, and hence vibration and noise, load distribution, thus requires some compromises by the designer.

2) The simulation results of the GTM on TE can effectively reflect the experimental results of GTM on vibration and noise. It means that the simulation analysis is helpful to understand the effects of GTM on the dynamic features and provides basic references for the future design of the gears with good dynamic performance and long service life before the prototype is available for the test.

\section{Acknowledgements}

The support for this research under Chinese National Key Technology Research and Development Program (Grant No. 2014BAF08B01) is gratefully acknowledged. Without their financial support, this work would not have been possible.

\section{References}

[1] Wu X, Gao Q and Li Z, "Mathematical and Finite Element Modeling of Micro-Modification for Marine Gear", International Journal of Rotating Machinery, (2015), pp. 1-7.

[2] Bruyère $\mathrm{J}, \mathrm{Gu} \mathrm{X}$ and Velex $\mathrm{P}$, "On the Analytical Definition of Profile Modifications Minimising Transmission Error Variations in Narrow-Faced Spur Helical Gears", Mechanism and Machine Theory, no. 92, (2015), pp. 257-272.

[3] Kuo Jao Huang and Ching Ya Su, "An investigation on helical gear pair stresses incorporating misalignment and detail modification”, Journal of Vibroengineering, vol. 15, no. 1, (2013), pp. 90-99.

[4] Ma H., Pang X., Feng R., and Wen B., "Evaluation of Optimum Profile Modification Curves of Profile Shifted Spur Gears Based on Vibration Responses", Mechanical Systems and Signal Processing, no. 70, (2016), pp. 1131-1149. 
[5] Li Runfang, "Modification Method and Stiffness Analysis of Gear Drives", Chongqing University Press, Chongqing, (1998).

[6] M. Platten, C. Blockley, B. James, S. Prabahakaran, and D. Scott, "Optimization of Gear Microgeometry for Durability and NVH", Sound \& Vibration, no. 4, (2009), pp. 7- 9.

[7] A. Kahraman and G. W. Blankenship, "Effect of Involute Tip Relief on Dynamic Response of Spur Gear Pairs", Journal of Mechanical Design, vol. 121, no. 2, (1999), pp. 313-315.

[8] Ruhai G, Xuyi J and Yang Wentao, "A Study on the Application of Micro-modifications on Gear Tooth Profile to Noise Reduction of Automotive Transmission", Automotive Engineering, vol. 31, no. 6, (2009), pp. 557-560.

[9] Wang C, Fang Z D and Zhang M L, "Analysis of Dynamics Behavior for Double Helical Gears Transmissions", Journal of Harbin Institute of Technology, vol. 43, no. 7, (2011), pp. 122-126.

[10] Chen S, Tang J and Wu L, "Dynamics Analysis of a Crowned Gear Transmission System with Impact Damping: Based on Experimental Transmission Error", Mechanism and Machine Theory, no. 74, (2014), pp.354-369.

[11] Howard I, Jia S. and Wang J., "The Dynamic Modelling of a Spur Gear in Mesh including Friction and a Crack", Mechanical Systems and Signal Processing, vol. 15, no. 5, (2001), pp. 831-851.

[12] Wagaj P and Kahraman A, "Influence of the Tooth Profile Modification on Helical Gear Durability", Journal of Mechanical Design, vol. 124, no. 3, (2002), pp. 501-510.

[13] Xiongxi $\mathrm{Wu}$, Qifeng Gao and Zesong Li, "Mathematical and Finite Element Modeling of MicroModification for Marine Gear", International Journal of Rotating Machinery, Article ID 902941, 7 pages, (2015).

[14] Liu G, Parker R G, "Dynamic Modeling and Analysis of Tooth Profile Modification for Multimesh Gear Vibration", Journal of Mechanical Design, vol. 130, no. 12, (2008), pp. 1-13.

[15] Xin Jingwei and Wang Shengze, "Research on the Gear's Modification and its Processing", Machinery, no. 5, (2009), pp. 19-21.

[16] TANG Jin-yuan, CHEN Xing-ming and LUO Cai-wang, "Contact Analysis of Spur Gears Based on Longitudinal Modification and Alignment Errors", Journal of Central South University, vol. 43, no. 5, (2012), pp. 1703-1709.

[17] Yang Sufen, He Jingliang and Dong Heyuan, "Exploration of Gear Elastic Deformation Modification based on MASTA", Journal of Mechanical Transmission, vol. 37, no. 4, (2013), pp. 33-36.

[18] Munro R G, Morrish L and Palmer D, "Gear Transmission Error Outside the Normal Path of Contact Due to Corner and Top Contact", Journal of Mechanical Engineering Science, vol. 213, no. 4, (1999), pp. 389-400.

[19] Oh, S., Oh, S., Kang, J., Lee, I., and Lyu, S, "A Study on Modeling and Optimization of Tooth Microgeometry for a Helical Gear Pair”, Int. J. Precis. Eng. Manuf., vol. 14, no. 3, (2013), pp. 423-427.

[20] Peng Guomin, Kang Liyun and Ren Haijun, "Analysis and Optimization for Gear Transmission Error", Automobile Technology, no. 12, (2009), pp. 95-99.

[21] Shi Ruonan, "Modification Design and Experimental Research of Automobile Transmission Gears", Taiyuan University of Technology, Taiyan, (2015).

[22] Shi Ruonan, Zhang Ruiliang, Wang Tie, Wu Zhifei and Liu Yaqiong. "A Study of Gear Elastic Deformation Modification based on Romax", Journal of Mechanical Transmission, vol. 39, no. 4, (2015), pp. $24-26+31$.

[23] Song Lemin, “Gear Profile and Gear Strength”, National Defense Industry Press, Beijing, (1987).

\section{Authors}
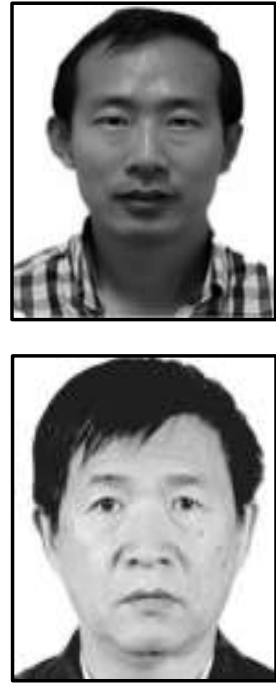

Ruiliang Zhang, He received his Ph.D. degree in Mechanical Engineering from the Taiyuan University of Technology, Taiyuan, China, in 2010. Now he works at University. His current research interests include gear transmission design, gear dynamics and fault diagnosis.

Tie Wang, He received his Ph.D. degree in Mechanical Engineering College from Beijing Institute of Technology, Beijing, China, in 2005. Now he works at Taiyuan University of Technology. His current research interests include mechanical transmission, dynamics and fault diagnosis. 